
\title{
BReserch S Suare \\ Quantum Correlated Heat Engine In XY Chainwith Dzyaloshinskii-Moriya Interactions
}

\section{Asadian}

University of Mohaghegh Ardabili

S. Ahadpour ( $\nabla$ ahadpour@uma.ac.ir)

University of Mohaghegh Ardabili

F. Mirmasoudi

University of Mohaghegh Ardabili

\section{Research Article}

Keywords: Quantum Correlation, Thermodynamic quantities, Dzyaloshinskii-Moriya, Heat engine.

Posted Date: December 6th, 2021

DOI: https://doi.org/10.21203/rs.3.rs-1131732/v1

License: (1) This work is licensed under a Creative Commons Attribution 4.0 International License.

Read Full License 


\title{
Quantum correlated heat engine in $X Y$ chain with Dzyaloshinskii-Moriya interactions
}

\author{
M. Asadian, S. Ahadpour ${ }^{*}$ F. Mirmasoudi ${ }^{\dagger}$ \\ December 1, 2021
}

Physics Department, University of Mohaghegh Ardabili, P.O. Box 179, Ardabil, Iran.

\begin{abstract}
In this paper, we consider a heat engines composed of two interactional qubits with spin-orbit interaction (Dzyaloshinskii-Moriya $(D M)$ ) subject to an external magnetic field, so that each qubit is considered as a cold or hot source. One intention of this work is to investigate the following question: is it possible the effects of $D M$ lead to improve basic thermodynamic quantities in this heat engine are coupled to local environments that are not necessarily at equilibrium? For this end, we investigate the effects of the temperature and the interaction rate of each qubit with its surrounding environment on quantum correlations such as quantum coherence and quantum discord and quantum entanglements, as well as the generated work. Finally we compare three quantum correlations (entanglement, discord, and coherence) with thermodynamic parameters and show that the output work is positive for what values of the magnetic field so that this cycle can be considered as a thermal machine.
\end{abstract}

Keywords: Quantum Correlation, Thermodynamic quantities, Dzyaloshinskii-Moriya, Heat engine.

\section{Introduction}

In recent years, there has been a revolution in the thermodynamic concepts, which is referred to as a new found among the physical theories. In this theory, the spread of information is described through quantum systems. This new theory, under the title of quantum thermodynamics, attempts to express thermodynamic concepts such as work, heat, efficiency, and so on by using quantum information theory [1-5]. A primary relation exists between thermodynamics and information theory. The laws of thermodynamics are a set of universal rules that explain the interaction between temperature, heat, work, energy, and entropy. The laws of thermodynamics are not only valid in the case of steam engines, but also for any other problem, including the sun, black holes, living things, and even the whole world. Thermodynamic rules are used only to express that a process is feasible or not. Quantum thermodynamics works to

\footnotetext{
*e-mail: ahadpour@uma.ac.ir

$\dagger$ e-mail: fmirmasoudi@uma.ac.ir
} 
develop quantum machines [6-9]. The most important application of quantum thermodynamics is quantum heat engines and one of the famous cycles in the quantum thermodynamic is the Carnot cycle [10-12]. The most common cyclic process is the Otto cycle [13-15]. The Otto cycle is the basis of all the heat engines performed based on the internal combustion process and hence it is of critical importance. In the following, we will begin to describe the basic processes (strokes) related to the classical form of the Otto cycle, in which the active material is a two-level system. The name of Otto cycle is retrieved from the German engineer Nicholas Otto, who worked for the manufacturing of the first four-stroke engine based on the initial design presented by Alphonse Beau de Rochas. Here, we want to discuss the role of quantum correlations in quantum thermodynamics [16]. First, Aliki defined the weak connection between heat and work in thermodynamics. He assumed that a change in the Hamiltonian and state of the sub-system is necessarily associated with the work and heat, respectively [17]. Afterward, more appropriate definitions are presented for thermodynamic work and heat [18-21]. Different quantum matters such as qubits [22-26], qudits [27-30], photons [31-33], harmonic oscillators [34-36], and so on are used in the field of heat engines. In this paper, a system is considered with two qubits a and $\mathrm{b}$ as the working mater. Consider an Otto cycle with the working matter in the form of a two-level system. According to the definition of work and quantum correlations for this system, we aim to examine the behavior and quantum correlations for our proposed model.

The paper is organized as follows. In Sec. 2, we give the model and calculate the density matrix for a system composed of two interactional qubits with spinorbit interaction in the Otto cycle. In Sec. 3, we define work and calculating the work, heat and efficiency of a heat cycle for our proposed model. In Sec. 4, we recall briefly entanglement, discord and coherence measures of the quantum correlations and we derive the analytical expression to the entanglement, discord and coherence and show how creating quantum correlations can be limited by the thermodynamics of the system. Finally, we give the conclusions in Section. 5 .

\section{MODEL SYSTEM: THE QUANTUM OTTO ENGINE}

Here we consider a system consisting of two interacting qubits $a$ and $b$ as the working material a four- level quantum Otto engine, which is described by $X Y$ Hamiltonian considering the spin-orbit interaction and magnetic field [37]:

$$
H=\left(J_{x} \sigma_{x_{a}} \sigma_{x_{b}}+J_{y} \sigma_{y_{a}} \sigma_{y_{b}}\right)+B\left(\sigma_{z_{a}}+\sigma_{z_{b}}\right)+D\left(\sigma_{x_{a}} \sigma_{y_{b}}-\sigma_{y_{a}} \sigma_{x_{b}}\right)
$$

where $\sigma_{[x, y, z]_{j}}$ denotes the Pauli operators that affect the qubits $j=a, b, J_{x}$ and $J_{y}$ are the strength of the antiferromagnetic couplings, $B$ is the intensity of the magnetic field, and $D$ is the spin-orbit interaction factor. We set $J_{x}=J_{y}=J$ and assume that the dynamics of the working matter density operator $\rho$ have the Markovian property, and can be stated by Lindblad master equation as follows [38]:

$$
\dot{\rho}=-[H, \rho]+\sum_{i} g_{i} L_{a_{i}}(\rho)
$$


Accordingly, $L_{a_{i}}=2 a_{i} \rho_{a_{i}}^{+}-\left\{a_{i}^{+} a_{i}, \rho\right\}$ and $a_{i}$ is the jump operator that describes the operation of the baths, and $g_{i}$ is the dissipation rate associated with the Lindblad term $L_{i}$ in the above equation. It is assumed that Lindblad operators of $L_{\sigma_{+}}^{j}$ with coefficient $g_{+}^{j}=\gamma_{j} \bar{n}_{j}$, and $L_{\sigma_{-}}^{j}$ with coefficient $g_{-}^{j}=\gamma_{j}\left(\bar{n}_{j}+1\right)$ are the jump operators in two general forms of raising operator $\sigma_{+}=|1\rangle\langle 0|$ and lowering operator $\sigma_{-}=|0\rangle\langle 1|$. The coefficients $\gamma_{j}$ is the interaction rate of each qubit with its surroundings and $\bar{n}_{j}$ is the population of the corresponding equilibrium temperature (the average particle number) which is defined by the following equation:

$$
\bar{n}_{j}=\left(e^{2 B_{j} / T_{j}}-1\right)^{-1}
$$

where $B_{j}$ and $T_{j}$ are the magnetic field and temperature for the bath coupled to qubit $j$, respectively. In the present paper, we consider different temperatures for the cold and hot baths coupled with two qubits. So, $\bar{n}_{C_{a}}$ and $\bar{n}_{C_{b}}$ are the population of the corresponding the temperature of the cold bath coupled with qubits $a$ and $b$ in the cooling process, and $\bar{n}_{H_{a}}$ and $\bar{n}_{H_{b}}$ are the population of the corresponding the temperature of the hot bath coupled with qubits $a$ and $b$ in the heating process. It is assumed that $\gamma_{a}$ and $\gamma_{b}$ (breakdown rates) take on different values. In this generalized Otto cycle, during the compression (expansion) stroke the magnetic field and spin-orbit interaction factor are changed, for both qubits, from $B_{1}$ to $B_{2}$ and $D_{1}$ to $D_{2}\left(B_{2}\right.$ to $B_{1}$ and $D_{2}$ to $\left.D_{1}\right)$ with $\mathrm{B}_{2}>\mathrm{B}_{1}$ and $\mathrm{D}_{2}>\mathrm{D}_{1}$. Under this condition, by following Ref [38] the density matrix can be expressed as,

$$
\rho=\frac{1}{\alpha}\left(\begin{array}{cccc}
r_{11} & 0 & 0 & 0 \\
0 & r_{22} & i r_{23} & 0 \\
0 & -i r_{23} & r_{33} & 0 \\
0 & 0 & 0 & r_{44}
\end{array}\right)
$$

where $r$ 's and $\alpha$ are reported in Appendix A. The working substance undergoes generalized a Otto cycle. This cycle consists of the following thermodynamic processes between the local equilibrium states of A, B, C and D [39] as follows:

1. Adiabatic compression (Isentropic) (A to B): The system is compressed, and work is done on the system. This stroke consists of volume and temperature variations, while entropy will remain constant throughout it. The material is isolated from the environment, and one of the Hamiltonian operators $J_{x}, J_{y}, B$ or $D$ changes accordingly to involve an increase in the energy gap.

2. Isochoric heating (B to $\mathrm{C}$ ): The temperature increases while the volume of the system (working substance) is constant. That means heat is absorbed from the source. During the evolution, the working matter proceeds to a steady state of Equation (2) with $\dot{\rho}=0$.

3. Adiabatic expansion (Isentropic) (C to D): The stroke power when a useful work comes out of the engine. Again, this stroke involves volume and temperature variations, and entropy is constant. The material is isolated from the environment, and one of the Hamiltonian parameters $J_{x}, J_{y}, B$ or $D$ gets its previous value. This leads to a decrease in the energy gap, which means approaching the classic case. 
4. Isochoric cooling (D to A): The environment is cooled in a constant volume and returns to its initial state, and it is ready to start the cycle again. To understand all aspects of the Otto-quantum cycle, we should examine how this four-stroke cycle is performed in the work environment which is a quantum system.

In the following, we will study the quantum work and three quantum correlations (entanglement, coherence, and discord) for this density matrix and compare the behavior of these three quantum correlations. We also present the variation of the work against different parameters.

\section{Work Definition}

In classical thermodynamics, we can define work as the potential energy of an external device that can be stored for later uses. In quantum systems, work is defined as a change in the energy of two systems [18]:

$$
W:=\operatorname{Tr}\left(H_{S_{B}} \rho_{S_{B}}\right)-\operatorname{Tr}\left(H_{S_{B}} U_{S_{B}} \rho_{S_{B}} U_{S_{B}}^{+}\right)
$$

where $U_{S_{B}}$ is a unitary continuous operator, $H_{S_{B}}$ is the Hamiltonian of the system and bath, and $\rho_{S_{B}}$ denotes the initial state. In this equation, $S$ and $B$ represent the system and bath, respectively. An important property in the Otto cycle is that it is isolated during the expansion and compression processes, and the change of energy is only in the form of external work. On the other hand, both work and heat variation may occur in cooling and heating processes and for unbalanced reservoirs. $-\mathrm{W}_{1}$ and $-\mathrm{W}_{2}$ represent the extracted work during the expansion and compression processes, and $\mathrm{Q}_{1}$ and $\mathrm{Q}_{2}$ are the heat obtained by the environment during heating and cooling processes. We assume $\mathrm{Q}>0$ when the heat is absorbed from the environment and the energy level increases in the system. Heat fluctuations cause the system to excite and the system leaves the ground state. Which means an increase in energy levels. So, we have for the cyclic evolution:

$$
W_{1}+W_{2}+Q_{1}+Q_{2}=0
$$

And the total derived work is equal to:

$$
W_{T}=-\left(W_{1}+W_{2}\right)=Q_{1}+Q_{2} .
$$

In this case, efficiency is defined as the ratio of the extracted work (if positive) to the total heat absorbed by the source [16]:

$$
\eta=\frac{W_{T}}{\sum_{Q_{i}>0} Q_{i}} .
$$

On condition $\bar{n}_{b}=0$, the followinng equations are obtaind. If $\rho^{C}$ and $\rho^{H}$ are the stable states for cold and hot baths with $r_{i j}^{C}$ and $r_{i j}^{H}$ elements, respectively, and if $\rho_{\tau}=U_{\tau} \rho_{S} U_{\tau}^{+}$, in this equation $\rho_{S}$ is the initial state of the system in the process and $\rho_{\tau}$ is the final state of the process where $U$ is a unitary operator. Now, the extracted work is equal to $\mathrm{W}=\operatorname{tr}\left(\rho_{\tau} H_{\text {fin }}\right)-\operatorname{tr}\left(\rho_{S} H_{\text {in }}\right)$, that $\mathrm{H}_{\text {in }}$ and $H_{\text {fin }}$ are the initial Hamiltonian with eigenvalues $E_{i}^{i n}$ and the final Hamiltonian with 
eigenvalues $E_{i}^{f i n}$, respectively. So for the heat absorbed from two hot sources, we have:

$$
Q_{1}=B_{2}\left(r_{11}^{C}-r_{11}^{H}+r_{44}^{H}-r_{44}^{C}\right) .
$$

Thus the total work is given by the following equation:

$$
W_{T}=\left(B_{2}-B_{1}\right)\left(r_{11}^{C}-r_{11}^{H}+r_{44}^{H}-r_{44}^{C}\right) .
$$

And the efficiency is defined as follows:
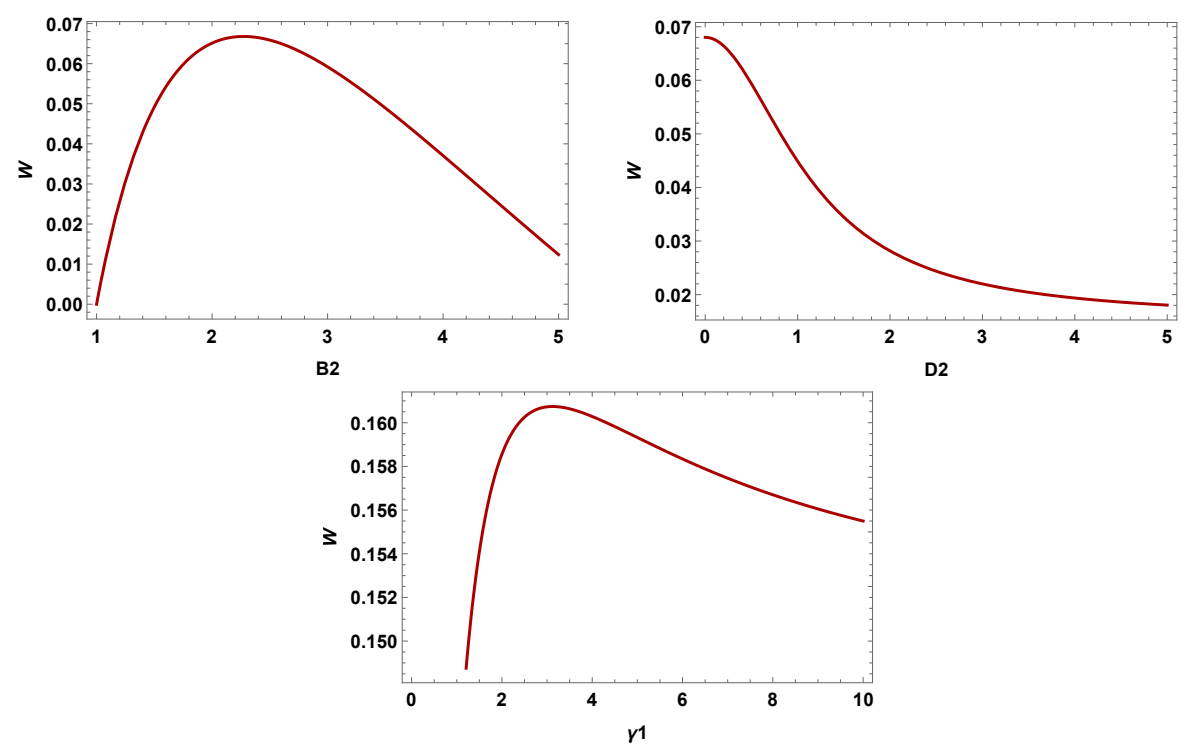

Figure 1: Plots of the total work for an Otto cycle operating between $T_{1}=1$ and $T_{2}=4$ using the steady state. (Top left): We fix $J=1, D=1$ and vary the magnetic field from $B_{1}=0$ to $B_{2}$ reported on the horizontal axis. (Top right): We fix $J=1, B_{1}=1$ and $B_{2}=2$; and vary the $D=0$ to $D=5$ reported on the horizontal axis. (Bottom): We fix $J=1, B_{1}=1, B_{2}=2, D=1, \gamma_{2}=5$ and we change $\gamma_{1}$ from 0 to the value reported on the horizontal axis.

$$
\eta=\frac{W_{T}}{Q_{1}}=1-\frac{B_{1}}{B_{2}} .
$$

It is straightforward to see that the efficiency only is under the effect of the magnetic field is identical on both spins can be controlled by it. But, the external work and heat not only depend on the magnetic field but also DzyaloshinskiiMoriya. We calculate the total work for different parameters such as the interaction rate of each qubit with its environment and the interaction factor between the spin-orbit and magnetic field. Based on Figure. 1, there is work for $\gamma_{1} \geq 1$ and it is maximum at $2 \leq \gamma_{1} \leq 3$. In Figure. 1, the output work is positive for $1 \leq B_{2} \leq 4$, so this idea can be implemented as a practical heat engine. Also we observed that the derived total work decreases by increasing the spin-orbit interaction factor. According to Figure. 2 that contour plots of the total work 

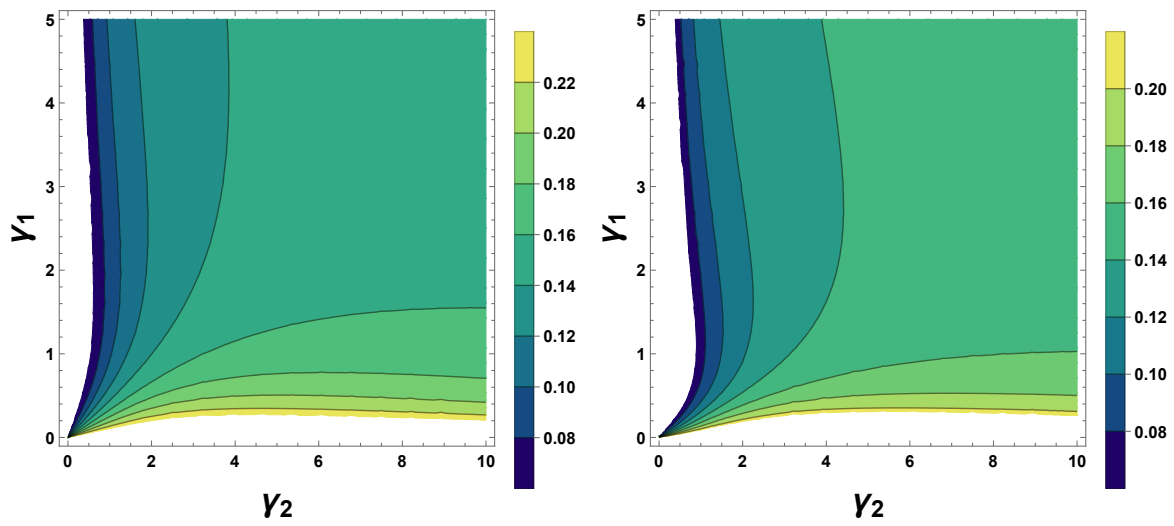

Figure 2: Contour plots of the total work for an Otto cycle corresponding to $n_{a}=1$ (left) and $n_{a}=2$ (right) as a function of $\gamma_{1}$ and $\gamma_{2}$, with $J=1, n_{b}=0$ and $D=1$ for both panels.

is ploted as a function $\gamma_{1}$ and $\gamma_{2}$. We find from Figure. 2, by fixing the $J$, if we keep $\gamma_{1}$ constant, by increasing the temperature, $W_{T}$ decreases. Considering the Dzyaloshinskii-Moriya interactions, despite the increase in the population of the corresponding equilibrum temperature, the total work is still there. From $\mathrm{Eq}(11)$, we see that increasing the magnetic field has a very positive effect on system efficiency. But increasing the temperature has no effect on efficiency, efficiency is sensitive to changes in magnetic field.

\section{Quantum correlations}

In this section, we discuss the quantum correlations in our system by analyzing the behavior of the entanglement, quantum discord and quantum coherence.

\subsection{Quantum Entanglement}

Entanglement is one of the important and confusing phenomena in the quantum world. The entanglement is the property shared between two or more systems showing correlations that cannot be described by classical physics, and this kind of correlation does not exist in the macroscopic world. Two particles may be very far from each other, but they can relate to each other, and whatever happens to one, it immediately causes a change in the other one. In quantum mechanics, the entanglement relates to quantum correlations that are rooted in the inseparable nature of the state vector of the quantum system [16, 40-42]. If $\rho$ is the density matrix of two qubits, so $\lambda_{i}$ represent the roots of matrix $\rho \tilde{\rho}$ where $\tilde{\rho}$ is defined as follows:

$$
\tilde{\rho}=\rho\left(\sigma_{y_{a}} \otimes \sigma_{y_{b}}\right) \rho^{*}\left(\sigma_{y_{a}} \otimes \sigma_{y_{b}}\right) .
$$

Also, the entanglement can be calculated by the following equation:

$$
C(\rho)=\max \left(0, \sqrt{\lambda_{1}}-\sqrt{\lambda_{2}}-\sqrt{\lambda_{3}}-\sqrt{\lambda_{4}}\right),
$$


where $\lambda_{1}>\lambda_{2}>\lambda_{3}>\lambda_{4}$.

Because our matrix is X-state, concurrence is obtained in a special case from equation(15),

$$
\begin{aligned}
& t_{1}=2\left(\sqrt{r_{23} \times r_{32}}-\sqrt{r_{11} \times r_{44}}\right), \\
& t_{2}=2\left(\sqrt{r_{14} \times r_{41}}-\sqrt{r_{22} \times r_{33}}\right),
\end{aligned}
$$

and

$$
C(\rho)=\max \left[0, t_{1}, t_{2}\right]
$$

In Figure. 3 contour plots of the concurrence is ploted as a function $\gamma_{1}$ and $\gamma_{2}$.
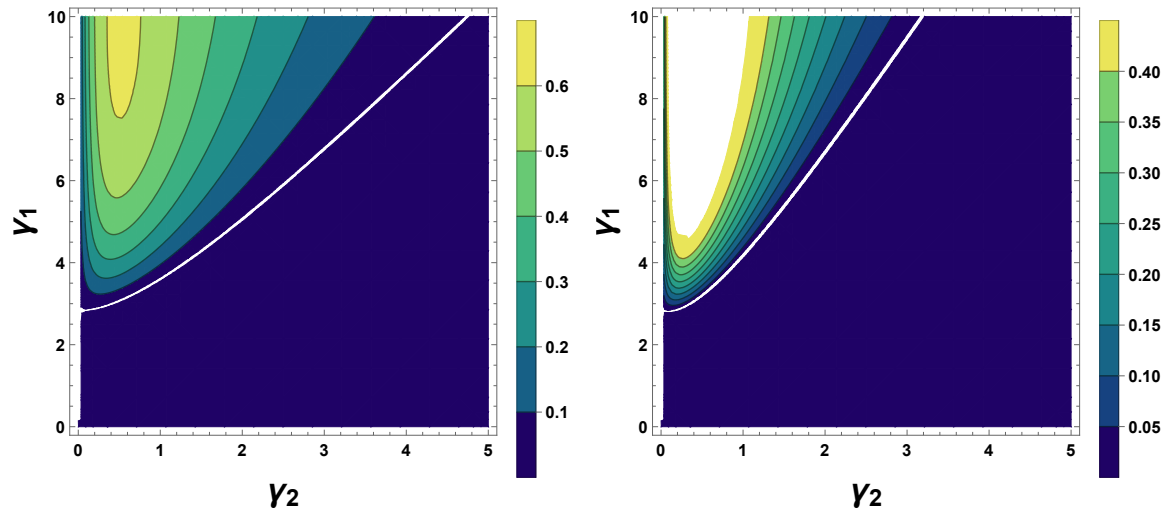

Figure 3: Contour plots of the concurrence of state $\rho$ in $\mathrm{Eq}(4)$ corresponding to $\bar{n}_{b}=0$ and $\bar{n}_{a}=1$ (left) and $\bar{n}_{a}=2$ (right) as a function of $\gamma_{1}$ and $\gamma_{2}$, with $J=1$ and $D=1$ for both panels.

We observe that the entanglement gets smaller by increasing the temperature. Even, the maximum amount of entanglement in this case is a small amount. We can see in Figure. 3 that the entanglement persists only for very low values of $\bar{n}$.

\subsection{Quantum Discord}

A bipartite quantum state consists of classical and conventional correlations. These correlations are jointly measured by their quantum mutual information which is a theoretical measure of the total correlation in a bipartite quantum state. In particular, if $\rho_{A B}$ is the density operator of a compound binary system $A B$ and $\rho_{A}\left(\rho_{B}\right)$ describes the density operator of term $A(B)$, thus the quantum mutual information is defined as [42],

$$
L(\rho(A B))=S\left(\rho_{A}\right)+S\left(\rho_{B}\right)-S\left(\rho_{A B}\right)
$$

where $S(\rho)=-\operatorname{tr}\left(\rho \log _{2} \rho\right)$ is the von Neumann entropy. Quantum mutual information may be written as the sum of the classical correlation $C\left(\rho_{A B}\right)$ and the quantum correlation $Q\left(\rho_{A B}\right)$ that is $L(\rho(A B))=C\left(\rho_{A B}\right)+Q\left(\rho_{A B}\right)$ [43-46]. This quantum term $Q(\rho)$ is called quantum discord [44]. This is a different kind of quantum correlation compared to the entanglement since the separable 
mixed modes (i.e., without entanglement) can have a nonzero quantum discord. Quantum discord is not always bigger than the entanglement [47, 48]. The quantum discord for the density matrix obtained from $\mathrm{Eq}(4)$ is obtained as follows [45]:

$$
D Q\left(\rho_{A B}\right)=\min \left(Q_{1}, Q_{2}\right),
$$

that

$$
\begin{gathered}
\epsilon_{1}=\frac{1}{2}\left[\left(r_{11}+r_{44}\right)+\sqrt{\left(r_{11}+r_{44}\right)^{2}+4\left|r_{14}\right|^{2}}\right], \\
\epsilon_{2}=\frac{1}{2}\left[\left(r_{11}+r_{44}\right)-\sqrt{\left(r_{11}+r_{44}\right)^{2}+4\left|r_{14}\right|^{2}}\right], \\
\epsilon_{3}=\frac{1}{2}\left[\left(r_{11}+r_{33}\right)+\sqrt{\left(r_{22}+r_{33}\right)^{2}+4\left|r_{23}\right|^{2}}\right], \\
\epsilon_{4}=\frac{1}{2}\left[\left(r_{22}+r_{33}\right)-\sqrt{\left(r_{22}+r_{33}\right)^{2}+4\left|r_{23}\right|^{2}}\right], \\
H(x)=-x \log _{2} x-(1-x) \log _{2}(1-x), \\
D_{1}=H\left(\frac{1+\sqrt{\left[1-2\left(r_{33}+r_{44}\right]^{2}+4\left(\left|r_{14}\right|+\left|r_{23}\right|\right)^{2}\right.}}{2}\right), \\
D_{2}=\sum_{i} r_{i i} \log _{2} r_{i i}-H\left(r_{11}+r_{33}\right), \\
Q_{j}=H\left(r_{11}+r_{33}\right)+\sum_{i=1}^{4} \epsilon_{i} \log _{2} \epsilon_{i}+D_{j} .
\end{gathered}
$$

In Figure. 4 contour plots of the quantum discord is ploted as a function $\gamma_{1}$
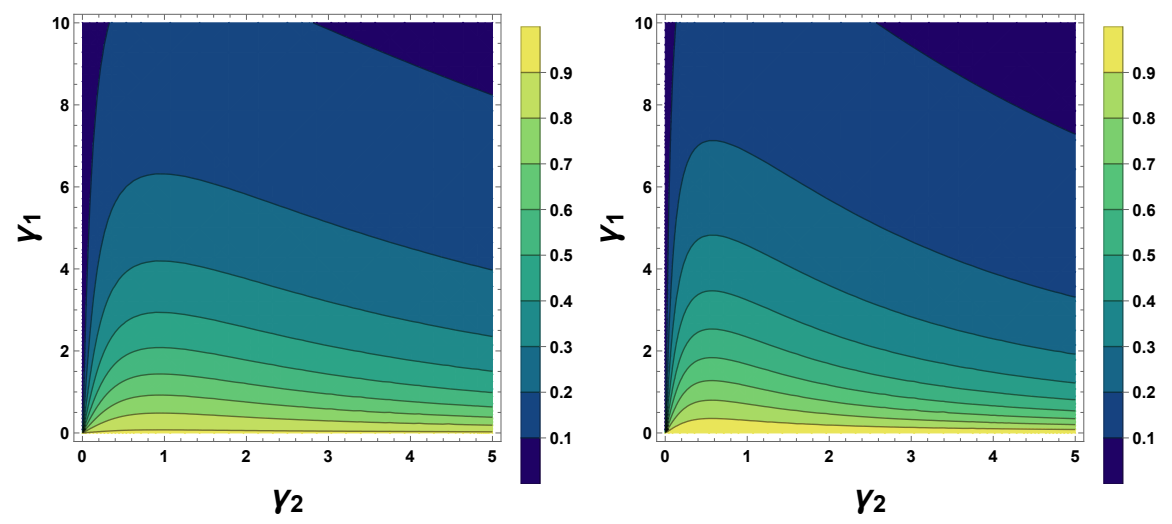

Figure 4: Contour plots of the discord of state $\rho$ in Eq: 4 corresponding to $\bar{n}_{b}=0$ and $\bar{n}_{a}=1$ (left) and $\bar{n}_{a}=2$ (right) as a function of $\gamma_{1}$ and $\gamma_{2}$, with $J=1$ and $D=1$ for both panels.

and $\gamma_{2}$. We find from Figure. 4 which quantum discord is sensitive to reservoir 
temperatures. It is important to note under what the condition quantum discord can be sustain more. Moreover, by compareing Figure. 3 and Figure. 4 we can find with increasing the interaction rate of any qubit with its environment, the entanglement becomes more tangible and the quantum discord decreases accordingly.

\subsection{Quantum Coherence}

The quantum coherence derived from the superposition of quantum states differentiates quantum from classical mechanics. The quantum coherence contained between different parts of quantum systems is the basis for the creation of quantum correlations [49-51]. The quantum coherence, which arises from the superposition principle, is one of the best non-classical features of a quantum system. A fundamental definition of quantum coherence is based on the non-diagonal elements of any quantum state. This relation is expressed by the following equation:

$$
\xi(t)=\sum_{i, j(i \neq j)}\left|\rho_{i j}(t)\right|
$$

where $\rho_{i j}(t),(i \neq j)$ represent the non-diagonal elements of the density matrix. Based on the research works conducted in this field [52-54], quantum coherence is achieved through the following equation:

$$
I(\rho, k)=-\frac{1}{2} \operatorname{Tr}[\sqrt{\rho}, k]^{2}
$$

where $\rho$ is the quantum state density matrix, $k$ is an observable, and [, ] is the displacement. For the resulting density matrix, the quantum coherence is calculated as,

$$
U=1-\lambda_{\max }(\chi)
$$

where $\lambda_{\max }$ is the maximum eigenvalue of the $3 \times 3$ matrix $\chi$ with the elements $\chi_{i j}=\operatorname{Tr}\left\{\sqrt{\rho}\left(\sigma_{i_{A}} \otimes I_{B}\right) \sqrt{\rho}\left(\sigma_{j_{A}} \otimes I_{B}\right)\right\}$ and $\sigma_{i}(i=1,2,3)$ represent the Pauli matrices.

In Figure. 5 contour plots of the quantum coherence is ploted as a function $\gamma_{1}$ and $\gamma_{2}$. We find from Figure. 5 which quantum coherence is sensitive to reservoir temperatures. It is important to note under what the condition quantum coherence can be sustain more. We observe that by increasing the interaction rate of any qubit with its environment, the quantum coherence further increases compared to the entanglement (see Figure. 3, Figure. 4 and Figure. 5 ). As can be seen, the entanglement and quantum discord get their maximum value at $\gamma_{2}=0.5$, while the maximum value of the quantum coherence occurs at $\gamma_{2}=0.7$. Figure. 6 shows the plots of three quantum correlations and the total work, as a function spin-orbit interaction factor $(D)$. As we can see, for some values of $D$, although we do not have entanglement, we have a discord. When we can identify quantum correlations through the discord, we see that positive works happening in our cycle. And we also see that with increasing the spin-orbit interaction factor, the coherence increases. Therefore, the quantum coherence behavior in terms of the Dzyaloshinskii-Moriya interactions coefficient is the opposite of the total work behavior. 

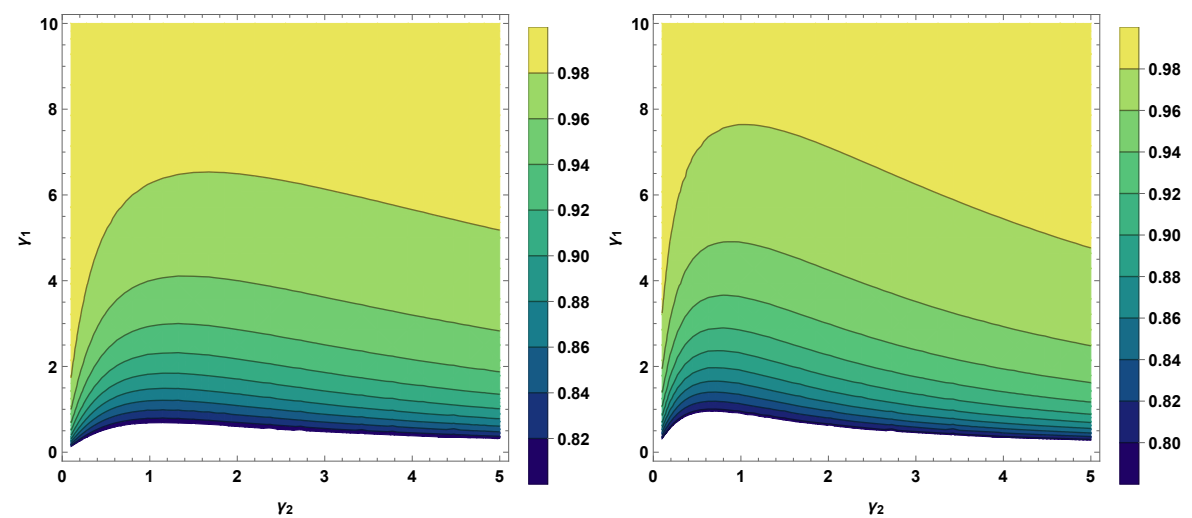

Figure 5: Contour plots of the coherence of state $\rho$ in Eq: (4) corresponding to $\bar{n}_{b}=0$ and $\bar{n}_{a}=1$ (left) and $\bar{n}_{a}=2$ (right) as a function of $\gamma_{1}$ and $\gamma_{2}$, with $J=1$ and $D=1$ for both panels.

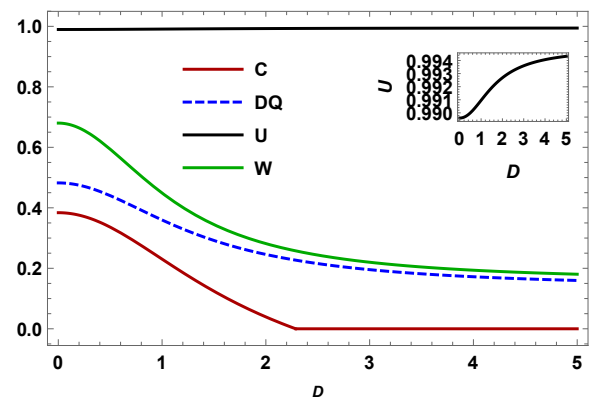

Figure 6: Plots of the total work, coherence, entanglement and discord for an Otto cycle operating between $T_{1}=1$ and $T_{2}=4$ using the steady state. We fix $J=1, B_{1}=1$ and $B_{2}=2$, and change $D$. 


\section{Conclusion}

In summary, we proposed a composite system composed of two interactional qubits with spin-orbit interaction. We discussed the work exteraction from the engine and its efficiency as well as the role of quantum correlations, characterized by the quantum entanglement, quantum discord and quantum coherence in the thermodynamical processes. The density matrix and quantum correlations are calculated for the state $X Y$, and we observe that the entanglement and the quantum coherence decrease and the quantum discord increases by increasing the population of the corresponding equilibrum temperature. Also, for constant $\gamma_{2}$, by increasing the $\gamma_{1}$, the entanglement and the total work become more tangible and the quantum discord decreases. But the quantum coherence further increases compared to the entanglement. In small $\gamma_{2}$, the quantum entanglement is maximum for large $\gamma_{1}$ but the total work is maximum for small $\gamma_{1}$. We also saw that, in the absence of entanglement in the system, the work done in the system is not necessarily zero, but discord can be a powerful tool for identifying positive work in the system. We have identified for what range of magnetic fields, we can consider this system as a heat engine. Our work show new light on the study of the work exteraction from the engine and its efficiency as well as the role of quantum correlations. 


\section{Appendix}

Here we provide the analytical formula for the steady state, (Eq: 4)

$$
\begin{aligned}
& \alpha=\left(\gamma_{1}+2 n_{1} \gamma_{1}+\gamma_{2}+2 n_{2} \gamma_{2}\right)^{2}\left(4\left(D^{2}+J^{2}\right)+\left(1+2 n_{1}\right)\left(1+2 n_{2}\right) \gamma_{1} \gamma_{2}\right), \\
& r_{11}=\frac{4 D^{2}\left(n_{1} \gamma_{1}+n_{2} \gamma_{2}\right)^{2}+4 J^{2}\left(n_{1} \gamma_{1}+n_{2} \gamma_{2}\right)^{2}}{\left(\gamma_{1}+2 n_{1} \gamma_{1}+\gamma_{2}+2 n_{2} \gamma_{2}\right)^{2}\left(4\left(D^{2}+J^{2}\right)+\left(1+2 n_{1}\right)\left(1+2 n_{2}\right) \gamma_{1} \gamma_{2}\right)} \\
& +\frac{n_{1} n_{2} \gamma_{1} \gamma_{2}\left(\gamma_{1}+2 n_{1} \gamma_{1}+\gamma_{2}+2 n_{2} \gamma_{2}\right)^{2}}{\left(\gamma_{1}+2 n_{1} \gamma_{1}+\gamma_{2}+2 n_{2} \gamma_{2}\right)^{2}\left(4\left(D^{2}+J^{2}\right)+\left(1+2 n_{1}\right)\left(1+2 n_{2}\right) \gamma_{1} \gamma_{2}\right)}, \\
& r_{22}=\frac{4 D^{2}\left(n_{1} \gamma_{1}+n_{2} \gamma_{2}\right)\left(\gamma_{1}+n_{1} \gamma_{1}+\gamma_{2}+n_{2} \gamma_{2}\right)}{\left(\gamma_{1}+2 n_{1} \gamma_{1}+\gamma_{2}+2 n_{2} \gamma_{2}\right)^{2}\left(4\left(D^{2}+J^{2}\right)+\left(1+2 n_{1}\right)\left(1+2 n_{2}\right) \gamma_{1} \gamma_{2}\right)} \\
& +\frac{4 J^{2}\left(n_{1} \gamma_{1}+n_{2} \gamma_{2}\right)\left(\gamma_{1}+n_{1} \gamma_{1}+\gamma_{2}+n_{2} \gamma_{2}\right)}{\left(\gamma_{1}+2 n_{1} \gamma_{1}+\gamma_{2}+2 n_{2} \gamma_{2}\right)^{2}\left(4\left(D^{2}+J^{2}\right)+\left(1+2 n_{1}\right)\left(1+2 n_{2}\right) \gamma_{1} \gamma_{2}\right)} \\
& +\frac{\left.n_{1}\left(1+n_{2}\right) \gamma_{1} \gamma_{2} \gamma_{1}+2 n_{1} \gamma_{1}+\gamma_{2}+2 n_{2} \gamma_{2}\right)^{2}}{\left(\gamma_{1}+2 n_{1} \gamma_{1}+\gamma_{2}+2 n_{2} \gamma_{2}\right)^{2}\left(4\left(D^{2}+J^{2}\right)+\left(1+2 n_{1}\right)\left(1+2 n_{2}\right) \gamma_{1} \gamma_{2}\right)}, \\
& r_{33}=\frac{4 D^{2}\left(n_{1} \gamma_{1}+n_{2} \gamma_{2}\right)\left(\gamma_{1}+n_{1} \gamma_{1}+\gamma_{2}+n_{2} \gamma_{2}\right)}{\left(\gamma_{1}+2 n_{1} \gamma_{1}+\gamma_{2}+2 n_{2} \gamma_{2}\right)^{2}\left(4\left(D^{2}+J^{2}\right)+\left(1+2 n_{1}\right)\left(1+2 n_{2}\right) \gamma_{1} \gamma_{2}\right)} \\
& +\frac{4 J^{2}\left(n_{1} \gamma_{1}+n_{2} \gamma_{2}\right)\left(\gamma_{1}+n_{1} \gamma_{1}+\gamma_{2}+n_{2} \gamma_{2}\right)}{\left(\gamma_{1}+2 n_{1} \gamma_{1}+\gamma_{2}+2 n_{2} \gamma_{2}\right)^{2}\left(4\left(D^{2}+J^{2}\right)+\left(1+2 n_{1}\right)\left(1+2 n_{2}\right) \gamma_{1} \gamma_{2}\right)} \\
& +\frac{\left(1+n_{1}\right) n_{2} \gamma_{1} \gamma_{2}\left(\gamma_{1}+2 n_{1} \gamma_{1}+\gamma_{2}+2 n_{2} \gamma_{2}\right)^{2}}{\left(\gamma_{1}+2 n_{1} \gamma_{1}+\gamma_{2}+2 n_{2} \gamma_{2}\right)^{2}\left(4\left(D^{2}+J^{2}\right)+\left(1+2 n_{1}\right)\left(1+2 n_{2}\right) \gamma_{1} \gamma_{2}\right)}, \\
& r_{44}=\frac{4 D^{2}\left(\gamma_{1}+n_{1} \gamma_{1}+\gamma_{2}+n_{2} \gamma_{2}\right)^{2}}{\left(\gamma_{1}+2 n_{1} \gamma_{1}+\gamma_{2}+2 n_{2} \gamma_{2}\right)^{2}\left(4\left(D^{2}+J^{2}\right)+\left(1+2 n_{1}\right)\left(1+2 n_{2}\right) \gamma_{1} \gamma_{2}\right)} \\
& +\frac{4 J^{2}\left(\gamma_{1}+n_{1} \gamma_{1}+\gamma_{2}+n_{2} \gamma_{2}\right)^{2}}{\left(\gamma_{1}+2 n_{1} \gamma_{1}+\gamma_{2}+2 n_{2} \gamma_{2}\right)^{2}\left(4\left(D^{2}+J^{2}\right)+\left(1+2 n_{1}\right)\left(1+2 n_{2}\right) \gamma_{1} \gamma_{2}\right)} \\
& +\frac{\left(1+n_{1}\right)\left(1+n_{2}\right) \gamma_{1} \gamma_{2}\left(\gamma_{1}+2 n_{1} \gamma_{1}+\gamma_{2}+2 n_{2} \gamma_{2}\right)^{2}}{\left(\gamma_{1}+2 n_{1} \gamma_{1}+\gamma_{2}+2 n_{2} \gamma_{2}\right)^{2}\left(4\left(D^{2}+J^{2}\right)+\left(1+2 n_{1}\right)\left(1+2 n_{2}\right) \gamma_{1} \gamma_{2}\right)}, \\
& r_{23}=\frac{2(D-i J)\left(n_{1}-n_{2}\right) \gamma_{1} \gamma_{2}}{\left(\gamma_{1}+2 n_{1} \gamma_{1}+\gamma_{2}+2 n_{2} \gamma_{2}\right)\left(4\left(D^{2}+J^{2}\right)+\left(1+2 n_{1}\right)\left(1+2 n_{2}\right) \gamma_{1} \gamma_{2}\right)} \text {, } \\
& r_{32}=\frac{2(D+i J)\left(n_{1}-n_{2}\right) \gamma_{1} \gamma_{2}}{\left(\gamma_{1}+2 n_{1} \gamma_{1}+\gamma_{2}+2 n_{2} \gamma_{2}\right)\left(4\left(D^{2}+J^{2}\right)+\left(1+2 n_{1}\right)\left(1+2 n_{2}\right) \gamma_{1} \gamma_{2}\right)}, \\
& r_{41}=r_{14}=r_{12}=r_{13}=r_{21}=r_{24}=r_{31}=r_{34}=r_{42}=r_{43}=0 \text {. }
\end{aligned}
$$

\section{Conflicts of interests}

M.A. developed the theoretical formalism, performed the analytic calculations and performed the numerical simulations. Both F.M and S.A. authors contributed to the final version of the manuscript. S.A. supervised the project.

\section{References}

[1] Allahverdyan AE, Balian R, Nieuwenhuizen TM. Maximal work extraction from finite quantum systems. EPL (Europhysics Letters). 2004;67(4):565.

[2] Quan H, Zhang P, Sun C. Quantum-classical transition of photonCarnot engine induced by quantum decoherence. Physical Review E. 2006;73(3):036122. 
[3] Watanabe G, Venkatesh BP, Talkner P, Del Campo A. Quantum performance of thermal machines over many cycles. Physical review letters. 2017;118(5):050601.

[4] Giorgi GL, Campbell S. Correlation approach to work extraction from finite quantum systems. Journal of Physics B: Atomic, Molecular and Optical Physics. 2015;48(3):035501.

[5] Haddadi S, Ghominejad M, Akhound A, Pourkarimi MR. Suppressing measurement uncertainty in an inhomogeneous spin star system. Scientific Reports. 2021;11(1):1-11.

[6] Latifah E, Purwanto A. Quantum heat engines; multiple-state 1d box system. 2013.

[7] Roßnagel J, Dawkins ST, Tolazzi KN, Abah O, Lutz E, Schmidt-Kaler F, et al. A single-atom heat engine. Science. 2016;352(6283):325-9.

[8] Niedenzu W, Mukherjee V, Ghosh A, Kofman AG, Kurizki G. Quantum engine efficiency bound beyond the second law of thermodynamics. Nature communications. 2018;9(1):1-13.

[9] Del Campo A, Goold J, Paternostro M. More bang for your buck: Superadiabatic quantum engines. Scientific reports. 2014;4(1):1-5.

[10] Roßnagel J, Abah O, Schmidt-Kaler F, Singer K, Lutz E. Nanoscale heat engine beyond the Carnot limit. Physical review letters. 2014;112(3):030602.

[11] Gardas B, Deffner S. Thermodynamic universality of quantum Carnot engines. Physical Review E. 2015;92(4):042126.

[12] Curzon FL, Ahlborn B. Efficiency of a Carnot engine at maximum power output. American Journal of Physics. 1975;43(1):22-4.

[13] Deffner S. Efficiency of harmonic quantum Otto engines at maximal power. Entropy. 2018;20(11):875.

[14] Kosloff R, Rezek Y. The quantum harmonic Otto cycle. Entropy. $2017 ; 19(4): 136$.

[15] Quan HT, Liu Yx, Sun CP, Nori F. Quantum thermodynamic cycles and quantum heat engines. Physical Review E. 2007;76(3):031105.

[16] Altintas F, Hardal AÜ, Müstecaplıoglu ÖE. Quantum correlated heat engine with spin squeezing. Physical Review E. 2014;90(3):032102.

[17] Alicki R. The quantum open system as a model of the heat engine. Journal of Physics A: Mathematical and General. 1979;12(5):L103.

[18] Goold J, Huber M, Riera A, Del Rio L, Skrzypczyk P. The role of quantum information in thermodynamics - a topical review. Journal of Physics A: Mathematical and Theoretical. 2016;49(14):143001.

[19] Vinjanampathy S, Anders J. Quantum thermodynamics. Contemporary Physics. 2016;57(4):545-79. 
[20] Kieu TD. The second law, Maxwell's demon, and work derivable from quantum heat engines. Physical review letters. 2004;93(14):140403.

[21] Quan H, Zhang P, Sun C. Quantum heat engine with multilevel quantum systems. Physical Review E. 2005;72(5):056110.

[22] Thomas G, Johal RS. Coupled quantum Otto cycle. Physical Review E. 2011;83(3):031135.

[23] Huang X, Xu H, Niu X, Fu Y. A special entangled quantum heat engine based on the two-qubit Heisenberg XX model. Physica Scripta. 2013;88(6):065008.

[24] Piloto AM, Ribeiro DS, Rodrigues SSM, Santos C, Santos JL, Sales MGF. Plastic antibodies tailored on quantum dots for an optical detection of myoglobin down to the femtomolar range. Scientific reports. 2018;8(1):111.

[25] Karimi B, Pekola J. Otto refrigerator based on a superconducting qubit: Classical and quantum performance. Physical Review B. 2016;94(18):184503.

[26] Huang X, Wang T, Yi X, et al. Effects of reservoir squeezing on quantum systems and work extraction. Physical Review E. 2012;86(5):051105.

[27] Altintas F, Müstecaplıŏlu ÖE. General formalism of local thermodynamics with an example: Quantum Otto engine with a spin- $1 / 2$ coupled to an arbitrary spin. Physical Review E. 2015;92(2):022142.

[28] Wang H, Liu S, He J. Thermal entanglement in two-atom cavity QED and the entangled quantum Otto engine. Physical Review E. 2009;79(4):041113.

[29] Barrios GA, Albarrán-Arriagada F, Cárdenas-López F, Romero G, Retamal J. Role of quantum correlations in light-matter quantum heat engines. Physical Review A. 2017;96(5):052119.

[30] Xiao G, Gong J. Construction and optimization of a quantum analog of the Carnot cycle. Physical Review E. 2015;92(1):012118.

[31] Scully MO, Zubairy MS, Agarwal GS, Walther H. Extracting work from a single heat bath via vanishing quantum coherence. Science. 2003;299(5608):862-4.

[32] Dillenschneider R, Lutz E. Energetics of quantum correlations. EPL (Europhysics Letters). 2009;88(5):50003.

[33] Türkpençe D, Altintas F, Paternostro M, Müstecaplioğlu ÖE. A photonic Carnot engine powered by a spin-star network. EPL (Europhysics Letters). 2017;117(5):50002.

[34] Zheng Y, Hänggi P, Poletti D. Occurrence of discontinuities in the performance of finite-time quantum Otto cycles. Physical Review E. 2016;94(1):012137. 
[35] Kosloff R, Rezek Y. The quantum harmonic Otto cycle. Entropy. 2017;19(4):136.

[36] Reid B, Pigeon S, Antezza M, De Chiara G. A self-contained quantum harmonic engine. EPL (Europhysics Letters). 2018;120(6):60006.

[37] Ahadpour S, Mirmasoudi F. Coupled two-qubit engine and refrigerator in Heisenberg model. Quantum Information Processing. 2021;20(2):1-13.

[38] Hewgill A, Ferraro A, De Chiara G. Quantum correlations and thermodynamic performances of two-qubit engines with local and collective baths. arXiv preprint arXiv:180610512. 2018.

[39] Peña FJ, Negrete O, Cortés N, Vargas P. Otto Engine: Classical and Quantum Approach. Entropy. 2020;22(7):755.

[40] Wootters WK. Entanglement of formation of an arbitrary state of two qubits. Physical Review Letters. 1998;80(10):2245.

[41] Horodecki R, Horodecki P, Horodecki M, Horodecki K. Quantum entanglement. Reviews of modern physics. 2009;81(2):865.

[42] Mishra MK, Maurya AK, Prakash H. Quantum discord and entanglement of quasi-Werner states based on bipartite entangled coherent states. International Journal of Theoretical Physics. 2016;55(6):2735-45.

[43] Dutta S, Adhikari B, Banerjee S. Quantum discord of states arising from graphs. Quantum Information Processing. 2017;16(8):1-17.

[44] Riedel CJ, Zurek WH. Quantum Darwinism in an everyday environment: Huge redundancy in scattered photons. Physical review letters. 2010;105(2):020404.

[45] Mirmasoudi F, Ahadpour S. Application quantum renormalization group to optimal dense coding in transverse Ising model. Physica A: Statistical Mechanics and its Applications. 2019;515:232-9.

[46] Ali M, Rau A, Alber G. Quantum discord for two-qubit X states. Physical Review A. 2010;81(4):042105.

[47] Luo S. Quantum discord for two-qubit systems. Physical Review A. 2008;77(4):042303.

[48] Luo S. Using measurement-induced disturbance to characterize correlations as classical or quantum. Physical Review A. 2008;77(2):022301.

[49] Gerry C, Knight P, Knight PL. Introductory quantum optics. Cambridge university press; 2005.

[50] Nielsen MA, Chuang I. Quantum computation and quantum information. American Association of Physics Teachers; 2002.

[51] Ahmad MA, Bukhari SH, Khan SN, Ran Z, Liao Q, Liu S. Nonclassical features of entangled coherent states. Journal of Modern Optics. $2011 ; 58(10): 890-5$. 
[52] Lei S, Tong P. Wigner-Yanase skew information and quantum phase transition in one-dimensional quantum spin- $1 / 2$ chains. Quantum Information Processing. 2016;15(4):1811-25.

[53] Behzadi N, Soltani E, Faizi E. Thermodynamic Cost of Creating Global Quantum Discord and Local Quantum Uncertainty. International Journal of Theoretical Physics. 2018;57(10):3207-14.

[54] Wu Z, Zhang L, Wang J, Li-Jost X, Fei SM. Uncertainty relations based on modified Wigner-Yanase-Dyson skew information. International Journal of Theoretical Physics. 2020;59(3):704-18. 